\title{
Induced current in the presence of magnetic flux tube of small radius
}

\author{
Alexander I. Milstein and Ivan S. Terekhov* \\ Budker Institute of Nuclear Physics, 630090 Novosibirsk, Russia
}

(Dated: February 19, 2018)

\begin{abstract}
The induced current density, corresponding to the massless Dirac equation in $(2+1)$ dimensions in a magnetic flux tube of small radius is considered. This problem is important for graphene. In the case, when an electron can not penetrate the region of nonzero magnetic field, this current is the odd periodical function of the magnetic flux. If the region inside the magnetic tube is not forbidden for penetration of electron, the induced current is not a periodical function of the magnetic flux. However in the limit $R \rightarrow 0$, where $R$ is the radius of magnetic flux tube, this function has the universal form which is independent of the magnetic field distribution inside the magnetic tube at fixed value of the magnetic flux.
\end{abstract}

PACS numbers: 03.65.Vf, 73.43.Cd, 81.05.Uw

\section{INTRODUCTION}

The Aharonov-Bohm effect predicted in Ref $\stackrel{1}{2}$ has been investigated in numerous papers, see review $\stackrel{2}{2}$. Due to this quantum effect, the vector potential affects the electron even in the case when the electron can not penetrate the region of non-zero magnetic field. This effect has been investigated within non-relativistic ${ }^{1,3}-\underline{5}$ and relativistic $\underline{3,4,6-\underline{8}}$ wave equations. In quantum field theory, similar effect has been investigated in Refs,$\underline{\underline{6}-13}$. One of the consequences of the Aharonov-Bohm effect is scattering of a charged particle in the field of an infinitesimally thin solenoid. It was shown in Refs, ${ }^{7,8}$ that the Dirac Hamiltonian in the background of the field of infinitesimally thin solenoid requires a self-adjoint extension. This extension can be parametrized by one extension parameter $\theta$. As a result, the eigen functions of the Hamiltonian and the corresponding scattering cross section depend on this parameter.

After recent fabrication of a monolayer graphite (graphene), Ref $\underline{14}$, new possibilities to study the Aharonov-Bohm effect have appeared. The single electron dynamics in graphene is described by the massless Dirac equation in $(2+1)$ dimensions, see Refs $\stackrel{15-18}{-18}$. Using the Green's function of this equation in the field of infinitesimally thin solenoid, the induced current density has been investigated analytically in Ref $\stackrel{19}{\underline{19}}$. Calculations have been performed exactly in a magnetic flux tube in the case when an electron can not penetrate the region of nonzero magnetic field. In the recent paper ${ }^{20}$, the local density of states in graphene was obtained in the field of infinitesimally thin solenoid.

In the present paper, we derive the induced current density, corresponding to the massless Dirac equation in $(2+1)$ dimensions, in the background of the magnetic flux tube of finite radius $R$. We investigate in detail the asymptotics $R \longrightarrow 0$ of this current density at fixed magnetic flux through the tube but for arbitrary distribution of a magnetic field inside the tube. We consider two cases. In the first case an electron can not penetrate the region of nonzero magnetic field, and in the second case this is allowed. Naturally, the result obtained in the first case is independent of the distribution of magnetic field inside the flux tube and coincides with the result of Ref $\stackrel{19}{\underline{19}}$. More interestingly, in the second case the induced current density is also independent of the magnetic field distribution at $R \longrightarrow 0$. In another words, we find the explicit value of the extension parameter $\theta$, Refs $\underline{\underline{8}, 11,12}$, and show that it is independent of the magnetic field distribution.

The article is organized as follows. In Sec. II we derive the Green's function of an electron in a magnetic field of the flux tube and obtain the induced current density at distances $r \gg R$. Calculation of the induced current density in the first and the second cases of the problem is performed in Sec. III and Sec. IV respectively. We conclude with a brief summary of our results.

\section{GENERAL DISCUSSION}

The induced current density, corresponding to the massless Dirac equation in $(2+1)$ dimensions in a magnetic field, has the form, Ref $\stackrel{19}{ }$ :

$$
\boldsymbol{j}_{\text {ind }}(\boldsymbol{r})=-i e \int_{C} \frac{d \epsilon}{2 \pi} \operatorname{Tr}\{\boldsymbol{\sigma} G(\boldsymbol{r}, \boldsymbol{r} \mid \epsilon)\},
$$

where the Green's function $G\left(\boldsymbol{r}, \boldsymbol{r}^{\prime} \mid \epsilon\right)$ obeys the equation

$$
[\epsilon-\boldsymbol{\sigma} \cdot(\boldsymbol{p}-e \boldsymbol{A}(\boldsymbol{r}))] G\left(\boldsymbol{r}, \boldsymbol{r}^{\prime} \mid \epsilon\right)=\delta\left(\boldsymbol{r}-\boldsymbol{r}^{\prime}\right) .
$$

Here $\boldsymbol{\sigma}=\left(\sigma_{x}, \sigma_{y}\right)$, and $\sigma_{i}$ are the Pauli matrices; $\boldsymbol{p}=$ $\left(p_{x}, p_{y}\right)$ is the momentum operator, $\boldsymbol{r}=(x, y), \boldsymbol{A}(\boldsymbol{r})$ is the vector potential of the magnetic field, $e$ is the electron charge, the system of units $\hbar=c=1$. According to the Feynman rules, the contour $C$ of integration over $\epsilon$ goes below the real axis in the left half plane and above the real axis in the right half plane of the complex $\epsilon$ plane. The magnetic field of the flux is $\boldsymbol{B}(r)=B(r) \boldsymbol{\nu}$, where $\boldsymbol{\nu}$ is the unit vector directed along $\mathrm{z}$ axis, $B(r)$ is a continuous function which obeys the condition $B(r)=0$ for $r>R$. It is convenient to choose the vector potential in the form

$$
\boldsymbol{A}(\boldsymbol{r})=\frac{\Phi[\boldsymbol{\nu} \times \boldsymbol{r}]}{2 \pi r} V(r),
$$


where $\Phi$ is the total magnetic flux through the tube, and a function $V(r)$ is

$$
V(r)=\frac{2 \pi}{\Phi r} \int_{0}^{r} d y y B(y)
$$

Using the analytical properties of the Green's function, we deform the contour of integration over $\epsilon$ in Eq. (11) so that it coincides with the imaginary axis. Thus it is necessary to find the function $G\left(\boldsymbol{r}, \boldsymbol{r}^{\prime} \mid i \epsilon\right)$ which we represent as

$$
G\left(\boldsymbol{r}, \boldsymbol{r}^{\prime} \mid i \epsilon\right)=[i \epsilon+\boldsymbol{\sigma} \cdot(\boldsymbol{p}-e \boldsymbol{A}(\boldsymbol{r}))] D\left(\boldsymbol{r}, \boldsymbol{r}^{\prime} \mid i \epsilon\right)
$$

where $\hat{D}\left(\boldsymbol{r}, \boldsymbol{r}^{\prime} \mid i \epsilon\right)$ is the Green's function of the squared Dirac equation

$$
\left[\epsilon^{2}+(\boldsymbol{p}-e \boldsymbol{A}(\boldsymbol{r}))^{2}-e \sigma_{z} B(r)\right] D\left(\boldsymbol{r}, \boldsymbol{r}^{\prime} \mid i \epsilon\right)=-\delta\left(\boldsymbol{r}-\boldsymbol{r}^{\prime}\right) .
$$

to Eq. (6), we obtain the following equations for the (6) functions $\mathcal{D}_{m}^{( \pm)}\left(r, r^{\prime} \mid i \epsilon\right)$

Substituting the function $D\left(\boldsymbol{r}, \boldsymbol{r}^{\prime} \mid i \epsilon\right)$ in the form

$$
\begin{aligned}
& D\left(\boldsymbol{r}, \boldsymbol{r}^{\prime} \mid i \epsilon\right)= \\
& \sum_{m=-\infty}^{\infty} \frac{e^{i m\left(\phi-\phi^{\prime}\right)}}{2 \pi}\left(\begin{array}{cc}
\mathcal{D}_{m}^{(+)}\left(r, r^{\prime} \mid i \epsilon\right) & 0 \\
0 & \mathcal{D}_{m}^{(-)}\left(r, r^{\prime} \mid i \epsilon\right)
\end{array}\right)
\end{aligned}
$$

and the $\delta$-function as

$$
\delta\left(\boldsymbol{r}-\boldsymbol{r}^{\prime}\right)=\frac{\delta\left(r-r^{\prime}\right)}{2 \pi \sqrt{r r^{\prime}}} \sum_{m=-\infty}^{\infty} e^{i m\left(\phi-\phi^{\prime}\right)}
$$

$$
\left[\frac{\partial^{2}}{\partial r^{2}}+\frac{1}{r} \frac{\partial}{\partial r}-\epsilon^{2}-\left(\frac{m}{r}-\gamma V(r)\right)^{2}+s_{ \pm} \frac{\gamma}{r} \frac{\partial(r V(r))}{\partial r}\right] \mathcal{D}_{m}^{( \pm)}\left(r, r^{\prime} \mid i \epsilon\right)=\frac{\delta\left(r-r^{\prime}\right)}{\sqrt{r r^{\prime}}}
$$

Here $s_{ \pm}= \pm 1, \gamma=\Phi / \Phi_{0}$, and $\Phi_{0}=2 \pi / e$ is the elementary magnetic flux. In the region $r, r^{\prime}>R$, the function $V(r)$ is $V(r)=1 / r$ so that the term with $s_{ \pm}$in Eq. (9) vanishes. As a result, the general solution of Eq. (9) in this region has the form

$$
\mathcal{D}_{m}^{( \pm)}\left(r, r^{\prime} \mid i \epsilon\right)=-\left(I_{|\lambda|}\left(|\epsilon| r_{<}\right) K_{|\lambda|}\left(|\epsilon| r_{>}\right)+\beta_{m}^{( \pm)} K_{|\lambda|}(|\epsilon| r) K_{|\lambda|}\left(|\epsilon| r^{\prime}\right)\right), \quad r, r^{\prime}>R
$$

Here $I_{\alpha}(x)$ and $K_{\alpha}(x)$ are the modified Bessel functions of the first and third kind, respectively, $r_{>}=\max \left(r, r^{\prime}\right)$, $r_{<}=\min \left(r, r^{\prime}\right)$, and $\lambda=m-\gamma$. The coefficient in front of the first term in Eq. (10) follows from the matching condition for the functions $\mathcal{D}_{m}^{( \pm)}\left(r, r^{\prime} \mid i \epsilon\right)$ at $r=r^{\prime}$. The coefficients $\beta_{m}^{( \pm)}$depend on the distribution of the magnetic field in the tube. They can be found from the matching condition at $r=R$ for the solutions (10) and the solutions of Eq. (9) in the region $r<R$.

Substituting Eqs. (5) and (7) to Eq. (1), we arrive at the following representation for the induced current density at $r>R$

$$
\boldsymbol{j}_{\text {ind }}(\boldsymbol{r})=\frac{e[\boldsymbol{\nu} \times \boldsymbol{r}]}{4 \pi^{2} r} \sum_{m=-\infty}^{\infty} \int_{-\infty}^{\infty} d \epsilon\left[\frac{\lambda}{r}\left(\mathcal{D}_{m}^{(+)}\left(r, r^{\prime} \mid i \epsilon\right)+\mathcal{D}_{m}^{(-)}\left(r, r^{\prime} \mid i \epsilon\right)\right)-\frac{\partial}{\partial r}\left(\mathcal{D}_{m}^{(+)}\left(r, r^{\prime} \mid i \epsilon\right)-\mathcal{D}_{m}^{(-)}\left(r, r^{\prime} \mid i \epsilon\right)\right)\right]_{r^{\prime}=r} .
$$


Then we use the solution (10) for $\mathcal{D}_{m}^{( \pm)}\left(r, r^{\prime} \mid i \epsilon\right)$ and finally obtain

$$
\begin{aligned}
& \boldsymbol{j}_{\text {ind }}(\boldsymbol{r})=\boldsymbol{j}_{\text {ind }}^{(1)}(\boldsymbol{r})+\boldsymbol{j}_{\text {ind }}^{(2)}(\boldsymbol{r}), \\
& \boldsymbol{j}_{\text {ind }}^{(1)}(\boldsymbol{r})=-\frac{e[\boldsymbol{\nu} \times \boldsymbol{r}]}{\pi^{2} r^{2}} \sum_{m=-\infty}^{\infty} \lambda \int_{0}^{\infty} d \epsilon I_{|\lambda|}(|\epsilon| r) K_{|\lambda|}(|\epsilon| r)=\frac{e N v_{F}[\boldsymbol{\nu} \times \boldsymbol{r}]}{4 \pi r^{3}}\left(\frac{1}{2}-|\tilde{\gamma}|\right)^{2} \tan (\pi \tilde{\gamma}), \\
& \boldsymbol{j}_{\text {ind }}^{(2)}(\boldsymbol{r})=-\frac{e[\boldsymbol{\nu} \times \boldsymbol{r}]}{2 \pi^{2} r} \sum_{m=-\infty}^{\infty} \int_{0}^{\infty} d \epsilon \epsilon\left(\beta_{m}^{(+)} K_{|\lambda|+\mu}(|\epsilon| r)-\beta_{m}^{(-)} K_{|\lambda|-\mu}(|\epsilon| r)\right) K_{|\lambda|}(|\epsilon| r),
\end{aligned}
$$

where $\mu=\operatorname{sign}(\lambda)$, the quantity $\tilde{\gamma}$ is the fractional part of $\gamma,|\tilde{\gamma}|<1$. It is defined as $\tilde{\gamma}=\gamma-n$ for $\gamma>0$, and $\tilde{\gamma}=\gamma+n$ for $\gamma<0, n$ is the maximal integer number less than $|\gamma|$. The first contribution, $\boldsymbol{j}_{i n d}^{(1)}(\boldsymbol{r})$, coincides with the result of Ref $\underline{\underline{19}}$ for the induced current density in the field of infinitesimally thin solenoid. This result has been obtained under assumption that an electron can not penetrate the region of nonzero magnetic field. The contribution $\boldsymbol{j}_{i n d}^{(1)}(\boldsymbol{r})$ is a periodical function of the magnetic flux, it depends on the fractional part of the quantity $\gamma$. It is equal to zero at the integer values of $\gamma$ but also at half-integer values of this quantity. The contribution $\boldsymbol{j}_{\text {ind }}^{(2)}(\boldsymbol{r})$ depends on the radius $R$ and distribution of the magnetic field inside the magnetic tube through the coefficients $\beta_{m}^{( \pm)}$. These coefficients are the functions of $\epsilon$, $R, \gamma$, and $m$.

\section{THE CASE OF PURE AHARONOV-BOHM EFFECT}

In the pure Aharonov-Bohm effect, an electron can not penetrate the region $r<R$ of nonzero magnetic field.
The corresponding boundary condition is $\boldsymbol{j}_{\text {ind }}(\boldsymbol{R})=0$. In this case $\mathcal{D}_{m}^{(+)}\left(r, r^{\prime} \mid i \epsilon\right)=\mathcal{D}_{m}^{(-)}\left(r, r^{\prime} \mid i \epsilon\right)$ and we obtain from Eq. (11) for the induced current density

$$
\boldsymbol{j}_{\text {ind }}(\boldsymbol{r})=\frac{e[\boldsymbol{\nu} \times \boldsymbol{r}]}{\pi^{2} r^{2}} \sum_{m=-\infty}^{\infty} \lambda \int_{0}^{\infty} d \epsilon \mathcal{D}_{m}^{(+)}\left(r, r^{\prime} \mid i \epsilon\right)
$$

The boundary condition gives the following form of the coefficient $\beta_{m}^{(+)}$in Eq. (10)

$$
\beta_{m}^{(+)}=-I_{|\lambda|}(x) / K_{|\lambda|}(x), \quad x=|\epsilon| R .
$$

At $x \ll 1$, the coefficient $\beta_{m}^{(+)}$in the leading order in $x$ reads

$$
\beta_{m}^{(+)} \approx-\frac{2^{1-2|\lambda|}|\lambda|}{\Gamma^{2}(|\lambda|+1)} x^{2|\lambda|}
$$

Using this expression, we obtain the asymptotics of the term $\boldsymbol{j}_{\text {ind }}^{(2)}(\boldsymbol{r})$ in Eq. (14) at large distances $r \gg R$

$$
\boldsymbol{j}_{\text {ind }}^{(2)}(\boldsymbol{r})=-\frac{e[\boldsymbol{\nu} \times \boldsymbol{r}]}{2 \pi r^{3}} \sum_{m=-\infty}^{\infty} \lambda|\lambda| \frac{\Gamma(|\lambda|+1 / 2) \Gamma(2|\lambda|+1 / 2)}{\Gamma^{3}(|\lambda|+1)}\left(\frac{R}{2 r}\right)^{2|\lambda|} .
$$

The contribution $\boldsymbol{j}_{\text {ind }}^{(2)}(\boldsymbol{r})$ is equal to zero at integer and half-integer values of $\gamma$ due to cancelation between different terms. For other values of $\gamma$ we introduce the quantity $\tilde{\lambda}=\tilde{m}-\gamma$, where $\tilde{m}$ minimizes the value $|\gamma-m|$, and obtain $\boldsymbol{j}_{\text {ind }}(\boldsymbol{r})$ in the leading and next-to-leading order in $R / r$

$$
\boldsymbol{j}_{\text {ind }}(\boldsymbol{r})=\frac{e[\boldsymbol{\nu} \times \boldsymbol{r}]}{4 \pi r^{3}}\left[\left(\frac{1}{2}-|\tilde{\gamma}|\right)^{2} \tan (\pi \tilde{\gamma})+2 \tilde{\lambda}|\tilde{\lambda}| \frac{\Gamma(|\tilde{\lambda}|+1 / 2) \Gamma(2|\tilde{\lambda}|+1 / 2)}{\Gamma^{3}(|\tilde{\lambda}|+1)}\left(\frac{\tilde{R}}{2 r}\right)^{2|\tilde{\lambda}|}\right] .
$$

The leading term $\boldsymbol{j}_{\text {ind }}^{(1)}(\boldsymbol{r})$ tends to zero at $r \rightarrow \infty$ as $1 / r^{2}$, and the correction $\boldsymbol{j}_{\text {ind }}^{(2)}(\boldsymbol{r})$ diminishes as $(\tilde{R} / r)^{2|\tilde{\lambda}|} / r^{2}$.

\section{THE CASE WHEN AN ELECTRON CAN PENETRATE THE REGION OF NONZERO MAGNETIC FIELD}

In this section we assume that an electron can penetrate into the magnetic tube. Let us start with a sim- 
ple case of the magnetic field distribution of the form $B(r)=B_{0} \theta(R-r)$, where $\theta(x)$ is the step-function. In this case the function $V(r)$ reads

$$
V(r)=\frac{r}{R^{2}} \theta(R-r)+\frac{1}{r} \theta(r-R) .
$$

This function is continuous one at $r=R$ so that the matching conditions for the functions $\mathcal{D}_{m}^{( \pm)}\left(r, r^{\prime} \mid i \epsilon\right)$ at $r=$ $R$, following from Eq. (6), are

$$
\begin{aligned}
& \mathcal{D}_{m}^{( \pm)}\left(R-0, r^{\prime} \mid i \epsilon\right)=\mathcal{D}_{m}^{( \pm)}\left(R+0, r^{\prime} \mid i \epsilon\right) \\
& \left.\frac{\partial \mathcal{D}_{m}^{( \pm)}\left(r, r^{\prime} \mid i \epsilon\right)}{\partial r}\right|_{r=R-0}=\left.\frac{\partial \mathcal{D}_{m}^{( \pm)}\left(r, r^{\prime} \mid i \epsilon\right)}{\partial r}\right|_{r=R+0}
\end{aligned}
$$

The solution of Eq. (9) in the region $r<R, r^{\prime}>R$ has the form:

$$
\begin{aligned}
\mathcal{D}_{m}^{( \pm)}\left(r, r^{\prime} \mid i \epsilon\right)= & C_{m}^{( \pm)}\left(r^{\prime}\right) r^{|m|} \exp \left\{-\frac{|\gamma| r^{2}}{2 R^{2}}\right\} \\
& \times{ }_{1} F_{1}\left(a^{( \pm)},|m|+1, \frac{|\gamma| r^{2}}{R^{2}}\right), \\
a^{( \pm)}= & \frac{\epsilon^{2} R^{2}}{4|\gamma|}+\frac{|\gamma||m|-\gamma m+|\gamma|-s_{ \pm} \gamma}{2|\gamma|},
\end{aligned}
$$

where $C_{m}^{( \pm)}\left(r^{\prime}\right)$ are some functions which can be found from the conditions (21), ${ }_{1} F_{1}(a, b, x)$ is the confluent $\mathrm{Hy}$ pergeometric function. Using Eqs. (10), (21), and (22) we obtain for the coefficients $\beta_{m}^{( \pm)}$

$$
\begin{aligned}
& \beta_{m}^{( \pm)}=-\frac{\mathcal{F}\left[b I_{|\lambda|}(x)+x I_{|\lambda|+1}(x)\right]-2|\gamma| \mathcal{F}^{\prime} I_{|\lambda|}(x)}{\mathcal{F}\left[b K_{|\lambda|}(x)-x K_{|\lambda|+1}(x)\right]-2|\gamma| \mathcal{F}^{\prime} K_{|\lambda|}(x)}, \\
& \mathcal{F}={ }_{1} F_{1}\left(a^{( \pm)},|m|+1,|\gamma|\right), \\
& \mathcal{F}^{\prime}=\frac{a^{( \pm)}}{|m|+1}{ }_{1} F_{1}\left(a^{( \pm)}+1,|m|+2,|\gamma|\right), \\
& b=|\lambda|+|\gamma|-|m|, \quad x=|\epsilon| R .
\end{aligned}
$$

It follows from Eqs. (23) and (14) that the induced current density is the odd function of the parameter $\gamma$, which is a consequence of the Furry theorem. Below for simplicity we assume that $\gamma>0$.

Using Eqs.(23) and (14) it is easy to obtain the asymptotics of the term $\boldsymbol{j}_{\text {ind }}^{(2)}(\boldsymbol{r})$ at distances $r \gg R$. In this case, the main contribution to the integral over $\epsilon$ in Eq. (14) is given by the region $\epsilon \sim 1 / r$ so that $x=|\epsilon| R \ll 1$. The main contribution to the sum over $m$ in Eq. (14) is given by the term proportional to $\beta_{m}^{(+)}$with $m=[\gamma]$, where $[y]$ means the integer part of $y$. For this $m$ the asymtotics of the coefficient $\beta_{m}^{(+)}$at $x \ll 1$ is

$$
\beta_{m}^{(+)}=-\frac{2}{\pi} \sin (\pi \tilde{\gamma}) .
$$

Substituting this expression to Eq. (14) and taking the integral over $\epsilon$ we find

$$
\boldsymbol{j}_{\text {ind }}^{(2)}(\boldsymbol{r})=-\frac{e[\boldsymbol{\nu} \times \boldsymbol{r}]}{4 \pi r^{3}}\left(\frac{1}{2}-|\tilde{\gamma}|\right) \tan (\pi \tilde{\gamma}) .
$$

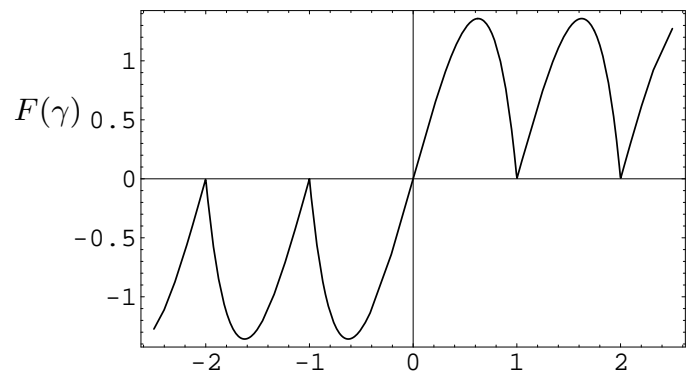

FIG. 1: Dependence of the function $F(\gamma)$, Eq. (26), on the $\gamma=e \Phi /(2 \pi c)$, where $\Phi$ is the magnetic flux.

Taking a sum of $\boldsymbol{j}_{\text {ind }}^{(1)}(\boldsymbol{r})$ and $\boldsymbol{j}_{\text {ind }}^{(2)}(\boldsymbol{r})$ we finally obtain

$$
\begin{aligned}
\boldsymbol{j}_{\text {ind }}(\boldsymbol{r}) & =\frac{e[\boldsymbol{\nu} \times \boldsymbol{r}]}{16 \pi r^{3}} F(\gamma), \\
F(\gamma) & =\left(1-4 \tilde{\gamma}^{2}\right) \tan (\pi \tilde{\gamma}) .
\end{aligned}
$$

The function $F(\gamma)$ is shown in Fig 1,

As it was shown in Ref $\frac{19}{19}$, the induced current in the case of pure Aharonov-Bohm effect is the periodical function of a magnetic flux. The reason is very simple. When an electron can not penetrate the region of nonzero magnetic field, the integer part of $\gamma$ can be eliminated from the wave equation by the gauge transformation. In opposite case considered in this section, we can not eliminate the integer part of $\gamma$. As a result, as it is seen from Eq. 25) and Fig,1, the induced current density is not a periodical function of the magnetic flux.

The result (26), obtained for the magnetic field $B(r)=$ $B_{0} \theta(R-r)$, coincides with the corresponding result of Ref $\frac{11}{11}$ for the extension parameter $\theta=\pi / 2$. Now we show that the leading asymptotics of the induced current density at $R / r \ll 1$ is independent of the distribution of magnetic field at the fixed magnetic flux. As it was pointed out above, at $R / r \ll 1$ the main contribution to the integral over $\epsilon$ in Eq. (14) is given by the region $\epsilon \sim 1 / r$ so that $|\epsilon| R \ll 1$. For $r, r^{\prime}>R$ the solution (10) is valid for any distribution of magnetic field. We use this solution for $r^{\prime}>r \geq R$. For $r^{\prime}>R \geq r$ we can neglect the term $\epsilon^{2}$ in Eq. (9) and write the equations for the functions $\mathcal{D}_{m}^{( \pm)}\left(r, r^{\prime} \mid i \epsilon\right)$ in the form:

$$
\begin{aligned}
& \hat{A}_{m} \hat{B}_{m} \mathcal{D}_{m}^{(+)}\left(r, r^{\prime} \mid i \epsilon\right)=0, \\
& \hat{B}_{m-1} \hat{A}_{m-1} \mathcal{D}_{m}^{(-)}\left(r, r^{\prime} \mid i \epsilon\right)=0, \\
& \hat{A}_{m}=\frac{\partial}{\partial r}+\frac{m+1}{r}-\gamma V(r), \\
& \hat{B}_{m}=\frac{\partial}{\partial r}-\frac{m}{r}+\gamma V(r) .
\end{aligned}
$$

The regular at $r=0$ solutions of the Eq. (27) have the form 


$$
\begin{aligned}
& \mathcal{D}_{m}^{(+)}\left(r, r^{\prime} \mid i \epsilon\right)=\zeta_{m}^{(+)}\left(r^{\prime}\right) r^{m} \exp \left\{-\gamma \int_{0}^{r} V(y) d y\right\} \begin{cases}1, & \\
\int_{0}^{r} x^{-(2 m+1)} \exp \left\{2 \gamma \int_{0}^{x} V(y) d y\right\} d x, & m<0\end{cases} \\
& \mathcal{D}_{m}^{(-)}\left(r, r^{\prime} \mid i \epsilon\right)=\zeta_{m}^{(-)}\left(r^{\prime}\right) r^{-m} \exp \left\{\gamma \int_{0}^{r} V(y) d y\right\} \begin{cases}\int_{0}^{r} x^{2 m-1} \exp \left\{-2 \gamma \int_{0}^{x} V(y) d y\right\} d x, & m>0 \\
1, & m \leq 0\end{cases}
\end{aligned}
$$

Here $\zeta_{m}^{( \pm)}\left(r^{\prime}\right)$ are some functions of $r^{\prime}$. Substituting the solutions (10), (28), and (29) to the matching condition (21) and using the asymptotics of the functions $I_{\lambda}(x)$ and $K_{\lambda}(x)$ at small $x$ we obtain the coefficients $\beta_{m}^{( \pm)}$. In the limit $|\epsilon| R \rightarrow 0$, only $\beta_{[\gamma]}^{(+)}$does not vanish and coincides with the result obtained for the magnetic field $B(r)=B_{0} \theta(R-r)$, see Eq. (24). Thus the leading asymptotics of the induced current density is independent of the distribution of the magnetic field in the magnetic flux tube. In our consideration we do not use any assumptions concerning the value of magnetic flux.

\section{CONCLUSION}

We have investigated the induced current density, corresponding to the massless Dirac equation in $(2+1)$ dimensions in a magnetic flux tube of small radius. This problem is important for graphene for which all formulas for the induced current density obtained in our pa- per should be multiplied by the factor $4 v_{F}$, the Fermi velocity is $v_{F} \approx 10^{6} \mathrm{~m} / \mathrm{s} \approx c / 300$. In the case of pure Aharonov-Bohm effect, when an electron can not penetrate the region of nonzero magnetic field, this current is the odd periodical function of the magnetic flux. In the opposite case, when the region inside the magnetic tube is not forbidden for penetration of electron, the induced current is not a periodical function of the magnetic flux. However in the limit $R \rightarrow 0$ this function has the universal form which is independent of the magnetic field distribution inside the magnetic tube at fixed value of the magnetic flux. Thus it is shown that the extension parameter $\theta$ introduced in the previous papers has the universal value $\pi / 2$. In the same way it is easy to show that the wave functions corresponding to the massless Dirac equation in $(2+1)$ dimensions in a magnetic flux tube have also the universal form in the limit $R \rightarrow 0$.

We are grateful to R.N. Lee for valuable discussions. The work was supported in part by RFBR (grant No 09-02-00024) and the foundation "Dynasty."
* A.I.Milstein@inp.nsk.su, I.S.Terekhov@inp.nsk.su

1 Y. Aharonov, D. Bohm, Phys. Rev. 115, 485 (1959).

2 M. Peskin, A. Tonomura, The Aharonov-Bohm Effect, Springer-Verlag, Berlin, 1998.

3 C.R. Hagen, Phys. Rev. Lett. 64, 503 (1990).

${ }^{4}$ V.R. Khalilov, Choon-Lin Ho, Ann. Phys. 323, 1280 (2008).

5 V.R. Khalilov, I.V. Mansurov, Lee Ki Eun, arXiv:10022826v1.

6 M.G. Alford, F. Wilczek, Phys. Rev. Lett. 62, 1071 (1989).

7 P. Gerbert, R. Jackiw, Commun. Math. Phys. 124, 229 (1989).

8 P. Gerbert, Phys. Rev. D 40, 1346 (1989).

9 S. Deser, R. Jackiw, and S Templeton, Ann. Phys. 140, 372 (1982).

10 M.G. Alford, J. March-Russell, F. Wilczek, Nucl. Phys. B 328, 140 (1989).

11 Yu.A. Sitenko, Ukr. J. Phys. 43, 1513 (1998).

12 Yu.A. Sitenko, Phys. Rev. D 60, 125017 (1999).

13 J.-B. Yau, E.P. De Poortere, and M. Shayegan, Phys. Rev. Lett. 88, 146801 (2002).

${ }^{14}$ K. S. Novoselov et al., Science 306, 666 (2004).
15 P.R. Wallace, Phys. Rev. 71, 622 (1947).

16 P.R. Wallace, Phys. Rev. 104, 666 (1956).

17 G. Semenoff, Phys. Rev. Lett. 53, 2449 (1984).

18 J. Gonzàlez, F. Guinea, and M. A. H. Vozmediano, Nucl. Phys. B 406, 771 (1993); 424, 595 (1994).

19 R. Jackiw, A.I. Milstein, S.-Y. Pi, and I.S. Terekhov, Phys. Rev. B 80, 033413 (2009).

20 A.O. Slobodeniuk, S.G. Sharapov, and V.M.Loktev, Phys. Rev. B 82, 075316 (2010).

21 M. Huefner, F. Molitor, A. Jacobsen, A. Pioda, C. Stampfer, K. Ensslin, and T. Ihn, Phys. Status Solidi 246, 2756 (2009).

22 J. Wurm, M. Wimmer, H.U. Baranger, K.Richter, Semicond. Sci. Technol. 25, 034003 (2010).

23 M. Zarenia, J. Milton Pereira Jr., A. Chaves, F. M. Peeters, G. A. Farias, Phys. Rev. B 81, 045431 (2010)

24 J. Schelter, D. Bohr, B. Trauzettel, Phys. Rev. B 81, 195441 (2010).

25 L.D. Landau, E.M. Lifshitz, Quantum Mechanics. Nonrelativistic theory, Pergamon Press, $§ 132$. 